\title{
Dynamics and stability predictions of variable helix milling
}

\author{
Qizhi Xie \\ School of Aircraft Engineering \\ Nanchang Hangkong University \\ Nanchang, China \\ e-mail: qzxie@163.com
}

\begin{abstract}
In milling, it is important to research the stability lobes charts which determine stable regions where no chatter occurs. This paper presents the nonlinear model of milling considering the variable helix angle. Base on this model, an improved semi-discretization method is used to predict stability. Due to the delay between each flute varies along the axial depth of the tool in milling, the cutting tool is discrete into some axial layers to simplify calculate. The effects of variable helix angle and uniform helix angle on the corresponding stability charts are explored, it is revealed that many unstable islands arise in the low radial immersion ratio. However, as the radial immersion rates increase, variable helix angle has a little effect on stability. This fully shows that the helix angle plays an important impact for stability. Meantime this also confirms our proposed method can accurately capture unstable phenomenon at low radial immersion milling operations.
\end{abstract}

Keywords-stability; milling; semi-discretization; variable helix angle; radial immersion rate

\section{INTRODUCTION}

Chatter, one of the most significant factors affecting the performances of a machine tool, is characterized by violent self-excited vibrations between the cutter and the workpiece developed under large metal removal rates [1]. The occurrence of chatter can cause the rough surface end and dimensional inaccuracy of the workpiece, along with unacceptably loud noise levels and accelerated tool wear. In general, chatter is the severe limitations for high productivity in milling operations. In order to seek effective measures to predict the onset of these vibrations, the behavior of dynamics and stability are worth to research. In practice, the prevalent strategies are to avoid chatter by applying analyses that predict parameter domains of stable cutting. Through analyzing the relationship between the axial depths of cut and spindle speed to choose the optimum stable cutting parameters so that one gets maximization of chatter-free material removal rate.

Efforts to understand the dynamic cutting process and chatter have a long history. The scientists have achieved fruitful results in this area and various methods have been developed which may be used to overcome the problem of chatter. Altintas developed a stability method which leads to analytical determination of stability lobes directly in the frequency domain [2, 3]. This method, known as zeroorder approximation, can achieve reasonably accurate predictions. However, zero-order approximation cannot accurately predict the stability lobes at low radial immersion milling operations. Merdol proposed multi frequency solution to solve this issue [4]. The semidiscretization method (SDM) was employed to obtain stability charts by Insperger [5, 6]. The delayed terms are discretized while the undelayed terms are unchanged and the time periodic coefficients are approximated by piecewise constant functions. Bayly proposed that the stability of interrupted cutting can be estimated by Temporal Finite Element Analysis (TFEA) [7]. This method was based on introducing finite elements in the time domain. The displacement and velocity at the end of each element are set equal to the displacement and velocity at the beginning of the next element while the cutting edge is engaged. A full discretization method (FDM) based on the direct integration scheme for prediction of milling stability was presented by Ding [8]. The simulation results indicated that the proposed method had high computational efficiency.

For simplicity, the above research results neglected the effect of the helix angle. In comparison to the case of straight tooth, helix milling not only accords with practical situation, but also can have access to rich dynamic properties. Isolated islands in the stability charts were first shown to occur in interrupted turning operations by Szalai [9]. The similar phenomenon associated with the helix angle of the tool was reported by Zatarain [10]. They extended the multi-frequency solution for helix angle tools in milling processes, and received the flip lobes became closed curves separated by horizontal lines where the depth of cut equals a multiple of the helix pitch. Insperger investigated the dynamics and stability of milling operations with helical fluted tool in time domain [11]. It is shown that unstable flip islands arise in the stability lobe diagram due to the helical flutes of the tool.

Up to now, few previous researches focus on the variable helix milling. To the authors' knowledge, only Turner [12], Sims [13] and Yusoff [14] considered the cases of variable helix angle in their studies. Sims proposed the tool could be discrete into a number of axial layers to solve the problem of variable helix angle. Yusoff combined with SDM and differential evolution to optimise variable helix end milling tools for minimising chatter. Based on the results of the former research studies $[6,8$, 13], we proposed an improved SDM to analyze the dynamics and stability of variable helix milling. 


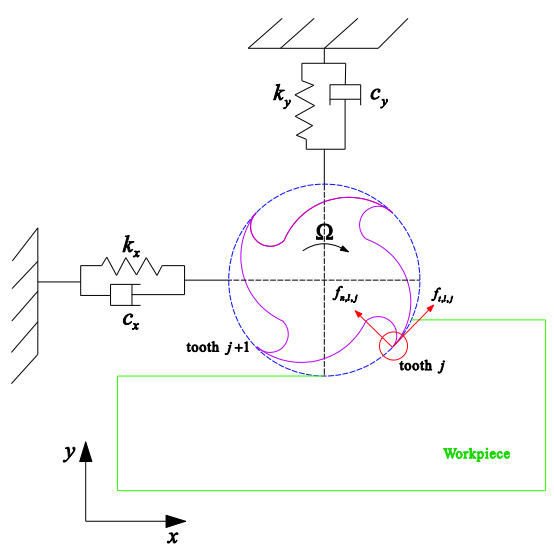

Figure 1. Schematic mechanical model of a two degree of freedom milling system

\section{MATHEMATICAL MODEL}

The mechanical model of the 2-DOF milling process is shown in the Fig .1. The tool is supposed to have flexibility in two orthogonal directions $(x, y)$. The mathematical equation of this model is given by:

$$
\boldsymbol{M} \ddot{\boldsymbol{x}}(t)+\boldsymbol{C} \dot{\boldsymbol{x}}(t)+\boldsymbol{K} \boldsymbol{x}(t)=\boldsymbol{F}(t),
$$

where the vector $\boldsymbol{x}(t)$ contains the displacement of the tool tip in the $x$ and $y$ directions, the matrices $\boldsymbol{M}, \boldsymbol{C}$ and $\boldsymbol{K}$ are the modal mass, damping and stiffness matrices, respectively. Due to the helix of the tool, the cutting force can be written as

$$
\boldsymbol{F}(t)=\left(\begin{array}{l}
F_{x}(t) \\
F_{y}(t)
\end{array}\right)=\left(\begin{array}{l}
\sum_{j=1}^{N} \sum_{l=1}^{L} f_{x, l, j}(t) \\
\sum_{j=1}^{N} \sum_{l=1}^{L} f_{y, l, j}(t)
\end{array}\right)
$$

where $N$ is the number of teeth, $L$ is the number of axial layer, the $x$ and $y$ components of the cutting force for the tooth $j$ on the layer $l$ at time $t$ are:

$$
\begin{aligned}
& f_{x, l, j}(t)=-f_{t, l, j}(t) \cos \left(\phi_{l, j}(t)\right)-f_{n, l, j}(t) \sin \left(\phi_{l, j}(t)\right) \\
& f_{y, l, j}(t)=-f_{t, l, j}(t) \sin \left(\phi_{l, j}(t)\right)-f_{n, l, j}(t) \cos \left(\phi_{l, j}(t)\right)
\end{aligned}
$$

The angular position of the $j$-th tooth of the $l$-th axial layer is defined by

$$
\phi_{l, j}(t)=\frac{2 \pi \Omega}{60} t+(j-1) \frac{2 \pi}{N}-z \frac{2 \tan \beta_{j}(l-1)}{d_{c} L},
$$

where $\Omega(\mathrm{rpm})$ is the spindle speed, $\beta_{j}$ is the helix angle of the $j$-th tooth, $d_{c}$ is the diameter of the tool and $z$ is the axial depth of cut. The tangential and the normal forces for the tooth $j$ on the layer $l$ can be expressed by

$$
\begin{aligned}
& f_{t, l, j}(t)=g\left(\phi_{l, j}(t)\right) K_{t} h_{l, j}(t) \Delta z, \\
& f_{n, l, j}(t)=g\left(\phi_{l, j}(t)\right) K_{n} h_{l, j}(t) \Delta z,
\end{aligned}
$$

where $\Delta z=z / L, K_{t}$ and $K_{n}$ are tangential and normal cutting coefficients, respectively. $g\left(\phi_{l, j}(t)\right)$ is the window function, it equals one if the tooth $j$ at layer $l$ is in the cut or zero when out of cut

$$
g\left(\phi_{l, j}(t)\right)= \begin{cases}1, & \text { if } \phi_{s t}<\phi_{j}(t)<\phi_{e x} \\ 0, & \text { Otherwise }\end{cases}
$$

Here, $\phi_{s t}$ and $\phi_{e x}$ define the angles at which the teeth enter and leave the workpiece. The dynamic chip thickness caused by vibration is

$$
\begin{aligned}
h_{l, j}(t)= & \left(x(t)-x\left(t-\tau_{l, j}\right)\right) \sin \left(\phi_{l, j}(t)\right) \\
& +\left(y(t)-y\left(t-\tau_{l, j}\right)\right) \cos \left(\phi_{l, j}(t)\right),
\end{aligned}
$$

where $\tau_{l, j}$ is the time delay that means the current tooth at the present time $t$ is to remove the surface waves that was generated at time $t-\tau_{l, j}$, and time delay can be described by

$$
\tau_{l, j}=T \frac{\Delta \phi_{l, j}}{2 \pi}
$$

where $T=60 / \Omega$ is time period, the pitch between one tooth and the next is given by

$$
\Delta \phi_{l, j}=\left\{\begin{array}{cl}
\phi_{l, j+1}(t)-\phi_{l, j}(t) & j<N, \\
\phi_{l, 1}(t)-\phi_{l, j}(t)+2 \pi & j=N .
\end{array}\right.
$$

According to the above analysis, the governing equation of milling process can be obtained. In the following, we will propose improved SDM to deal with variable helix milling issue.

\section{NUMERICAL ALGORITHMS}

Let $\quad \boldsymbol{q}(t)=(x(t), y(t))^{T} \quad, \quad \boldsymbol{p}(t)=\boldsymbol{M} \dot{\boldsymbol{q}}+\boldsymbol{C q} / 2 \quad$ and $\boldsymbol{y}(t)=(\boldsymbol{q}(t), \boldsymbol{p}(t))^{T}$. Through algebraic transformations, Eq. 1 can be represented by:

$$
\dot{\boldsymbol{y}}(\mathrm{t})=\boldsymbol{A}_{0} \boldsymbol{y}(t)+\sum_{j=1}^{N} \sum_{l=1}^{L}\left[\boldsymbol{A}_{l, j}(t) \boldsymbol{y}(t)-\boldsymbol{B}_{l, j}(t) \boldsymbol{y}\left(t-\tau_{l, j}\right)\right]
$$

with

$$
\boldsymbol{A}_{0}=\left(\begin{array}{cc}
-\boldsymbol{M}^{-1} \boldsymbol{C} / 2 & \boldsymbol{M}^{-1} \\
\boldsymbol{C} \boldsymbol{M}^{-1} \mathrm{C} / 4-\boldsymbol{K} & -\boldsymbol{C} \boldsymbol{M}^{-1} / 2
\end{array}\right)
$$

and $\boldsymbol{A}_{l, j}(t)=\boldsymbol{B}_{l, j}(t)=\left(\begin{array}{cccc}0 & 0 & 0 & 0 \\ 0 & 0 & 0 & 0 \\ h_{x x}(t) & h_{x y}(t) & 0 & 0 \\ h_{y x}(t) & h_{y y}(t) & 0 & 0\end{array}\right)$, where

$h_{x x}(t)=-g\left(\phi_{l, j}(t)\right)\left[K_{t} \cos \left(\phi_{l, j}(t)\right)+K_{n} \sin \left(\phi_{l, j}(t)\right)\right] \sin \left(\phi_{l, j}(t)\right) \Delta z$,

$h_{x y}(t)=-g\left(\phi_{l, j}(t)\right)\left[K_{t} \cos \left(\phi_{l, j}(t)\right)+K_{n} \sin \left(\phi_{l, j}(t)\right)\right] \cos \left(\phi_{l, j}(t)\right) \Delta z$

$h_{y x}(t)=-g\left(\phi_{l, j}(t)\right)\left[-K_{t} \sin \left(\phi_{l, j}(t)\right)+K_{n} \cos \left(\phi_{l, j}(t)\right)\right] \sin \left(\phi_{l, j}(t)\right) \Delta z$, 
The first step of semi-discretization is the construction of the time interval division of $\left[t_{i}, t_{i}+1\right]$ of length $\Delta t$, $i=0,1, \ldots$ so that $T=k \Delta t$, where $k$ is an integer that can be considered as an approximation parameter regarding the time period. The integers $m_{l, j}$ related to the delay $\tau_{l, j}$ can be approximately obtained by $m_{l, j}=\operatorname{int}\left(\left(\tau_{l, j}+\Delta t\right) / \Delta t\right)$, where int $(*)$ is the function that rounds positive numbers towards zero (e.g. int $(3.23)=3$ ). Since integer $m_{l, j}$ might be different for different semi-discretization steps, we introduce the maximum value of $m_{l, j}$ as $M=\max _{l, j=1,2, \ldots}\left\{m_{l, j}\right\}$.

Then solving (10) as an ordinary differential equation over the discretization period $\left[t_{i}, t_{i}+1\right]$ with initial condition $\boldsymbol{y}\left(t_{i}\right)=\boldsymbol{y}_{i}$, we can obtain:

$$
\begin{aligned}
& \boldsymbol{y}(t)=\mathrm{e}^{\boldsymbol{A}_{0}\left(t-t_{i}\right)} \boldsymbol{y}_{i}+\int_{t_{i}}^{t}\left\{\mathrm{e}^{\boldsymbol{A}_{0}(t-\xi)} \times\right. \\
& \left.\sum_{j=1}^{N} \sum_{l=1}^{L}\left[\boldsymbol{A}_{l, j}(\xi) \boldsymbol{y}(\xi)-\boldsymbol{B}_{l, j}(\xi) \boldsymbol{y}\left(\xi-\tau_{l, j}\right)\right]\right\} \mathrm{d} \xi .
\end{aligned}
$$

Equation (12) can be equivalently expressed as

$$
\begin{aligned}
& \boldsymbol{y}\left(t_{i+1}\right)=\mathrm{e}^{\boldsymbol{A}_{0} \Delta t} \boldsymbol{y}_{i}+\int_{t_{i}}^{t_{i+1}}\left\{\mathrm{e}^{\boldsymbol{A}_{0}\left(t_{i+1}-\xi\right)} \times\right. \\
& \left.\sum_{j=1}^{N} \sum_{l=1}^{L}\left[\boldsymbol{A}_{l, j}(\xi) \boldsymbol{y}(\xi)-\boldsymbol{B}_{l, j}(\xi) \boldsymbol{y}\left(\xi-\tau_{l, j}\right)\right]\right\} \mathrm{d} \xi,
\end{aligned}
$$

where $t_{i}=i \Delta t$ (with $i \in Z$ ) and

$$
\begin{aligned}
& \boldsymbol{A}_{l, j}(t)=\boldsymbol{A}_{i}^{<l, j>}+\frac{\boldsymbol{A}_{i+1}^{<l, j>}-\boldsymbol{A}_{i}^{<l, j>}}{\Delta t}\left(t-t_{i}\right), \\
& \boldsymbol{B}_{l, j}(t)=\boldsymbol{B}_{i}^{<l, j>}+\frac{\boldsymbol{B}_{i+1}^{<l, j>}-\boldsymbol{B}_{i}^{<l, j>}}{\Delta t}\left(t-t_{i}\right), \\
& \boldsymbol{y}(t)=\boldsymbol{y}_{i}+\frac{\boldsymbol{y}_{i+1}-\boldsymbol{y}_{i}}{\Delta t}\left(t-t_{i}\right), \\
& \boldsymbol{y}\left(t-\tau_{l, j}\right)=\boldsymbol{y}_{i-m_{l, j}}+\frac{\boldsymbol{y}_{i+1-m_{l, j}}-\boldsymbol{y}_{i-m_{l, j}}}{\Delta t}\left(t-t_{i}\right)
\end{aligned}
$$

with $\boldsymbol{A}_{i}^{<l, j>}=\boldsymbol{A}_{l, j}\left(t_{i}\right), \boldsymbol{B}_{i}^{<l, j>}=\boldsymbol{B}_{l, j}\left(t_{i}\right)$ and $\boldsymbol{y}_{i}=\boldsymbol{y}\left(t_{i}\right)$.

Substituting (14) into (13) leads to:

$$
\begin{aligned}
\boldsymbol{y}_{i+1}= & \left(\boldsymbol{\Phi}_{0}+\sum_{j=1}^{N} \sum_{l=1}^{L} F_{i}^{<l, j>}\right) \boldsymbol{y}_{i}+\sum_{j=1}^{N} \sum_{l=1}^{L} P_{i}^{<l, j>} \boldsymbol{y}_{i+1} \\
& -\sum_{j=1}^{N} \sum_{l=1}^{L} \boldsymbol{F}_{i}^{<l, j>} \boldsymbol{y}_{i-m_{l, j}}-\sum_{j=1}^{N} \sum_{l=1}^{L} \boldsymbol{P}_{i}^{<l, j>} \boldsymbol{y}_{i+1-m_{l, j}},
\end{aligned}
$$

where

$$
\begin{aligned}
\boldsymbol{F}_{i}^{<l, j>}= & \left(\boldsymbol{\Phi}_{1}-\frac{2}{\Delta t} \boldsymbol{\Phi}_{2}+\frac{1}{\Delta t^{2}} \boldsymbol{\Phi}_{3}\right) \boldsymbol{A}_{i}^{<l, j>} \\
& +\left(\frac{1}{\Delta t} \boldsymbol{\Phi}_{2}-\frac{1}{\Delta t^{2}} \boldsymbol{\Phi}_{3}\right) \boldsymbol{A}_{i+1}^{<l, j>}, \\
\boldsymbol{P}_{i}^{<l, j>}= & \left(\frac{1}{\Delta t} \boldsymbol{\Phi}_{2}-\frac{1}{\Delta t^{2}} \boldsymbol{\Phi}_{3}\right) \boldsymbol{A}_{i}^{<l, j>}+\left(\frac{1}{\Delta t^{2}} \boldsymbol{\Phi}_{3}\right) \boldsymbol{A}_{i+1}^{<l, j>} .
\end{aligned}
$$


ignored in the low radial immersion ratio. However, unstable islands will disappear as radial immersion ratio increases, helix angle almost no effect on milling stability (Fig .1(b)). These phenomena are in line with the conclusions of previous research [11].

To illustrate the proposed method on variable helix milling, the stability prediction charts are numerically studied at four different radial immersion ratios, i.e., 5\% and 10\% (Fig .3-Fig .4). Cutting force coefficients and modal parameters are selected as the same as above mentioned, but the values of helix angle have changed. It can be seen from Fig .2(a) and Fig .3 that variable helix milling will lead to stability significantly change in the low radial immersion ratio. In addition, many unstable islands also appeared in Fig .3(a1) and Fig .3(b1). We know that per tooth's helix angle has 10 degrees difference in these two charts, this is one of the reason lead to unstable islands appear in the low radial immersion ratio. However, compared with the Fig .2(a), the number of unstable island not increases in the Fig .3(a2) and Fig .3(b2). By comparing Fig .2(b) and Fig .4, it can be seen that variable helix angle basically no influence on stability in the large radial immersion ratio.
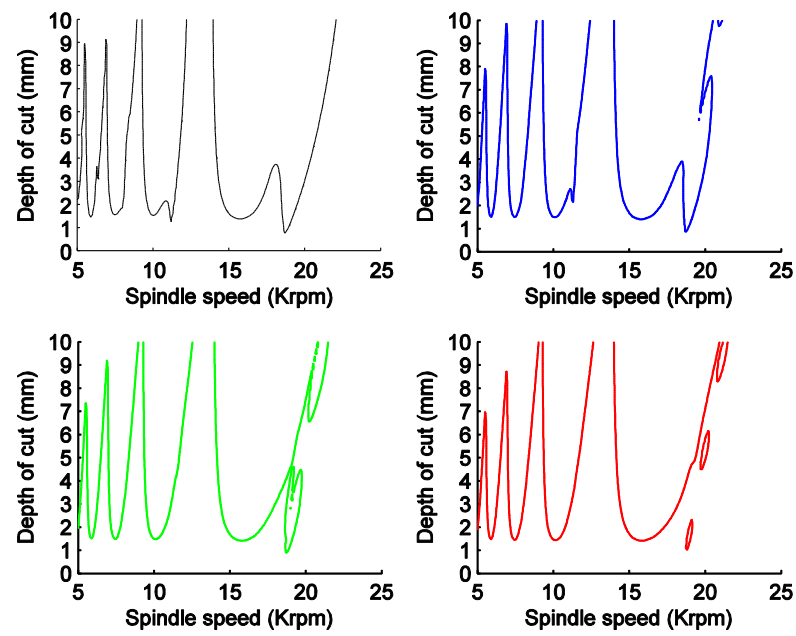

(a) Radial immersion ratio is 0.05
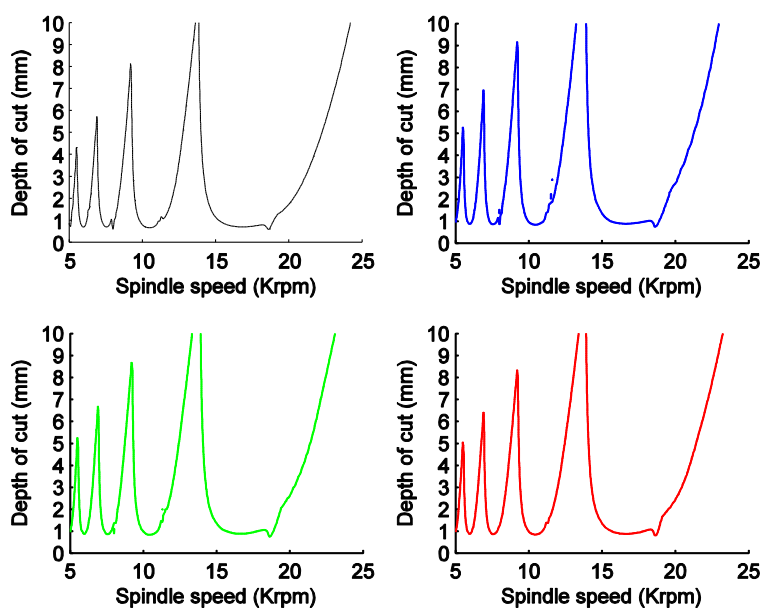

(b) Radial immersion ratio is 0.1

Figure 2. Stability charts for 2-DOF model: (-) $\beta=0$ 。, (-) $\beta=30$, $(-) \beta=45^{\circ}$ and $(-) \beta=60^{\circ}$ (a1) variable helix angle $\left(20^{\circ}, 30^{\circ}\right)$

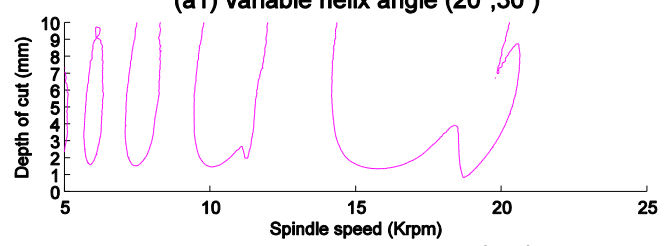

(a2) variable helix angle $\left(25^{\circ}, 30^{\circ}\right)$

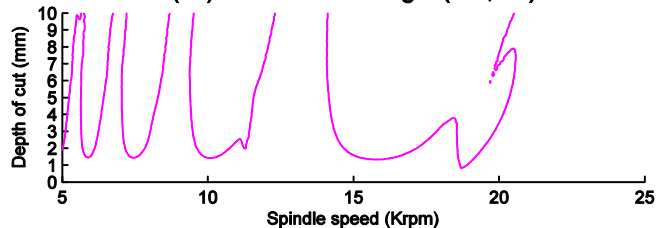

(b1) variable helix angle $\left(35^{\circ}, 45^{\circ}\right)$

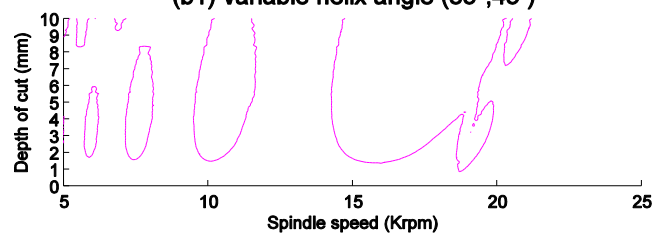

(b2) variable helix angle $\left(40^{\circ}, 45^{\circ}\right)$

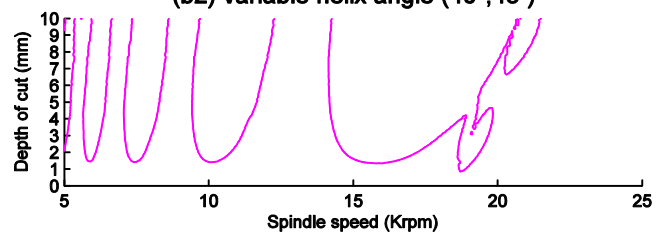

Figure 3. Stability charts for variable helix milling with radial immersion ratio $a_{d c}=0.05$

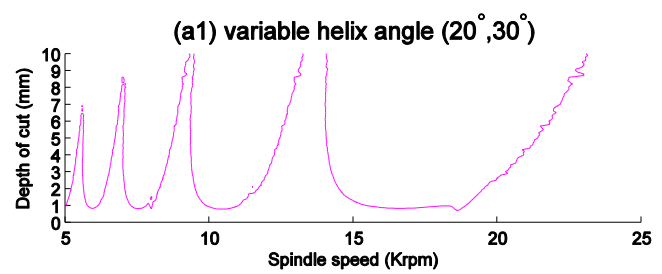

(a2) variable helix angle $\left(25^{\circ}, 30^{\circ}\right)$

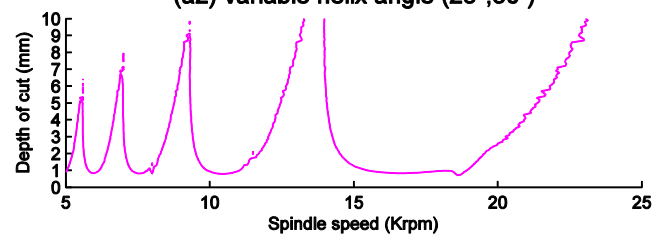

(b1) variable helix angle $\left(35^{\circ}, 45^{\circ}\right)$

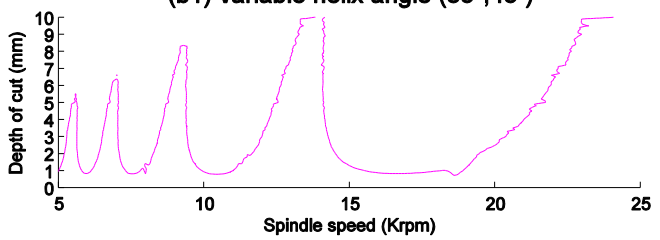

(b2) variable helix angle $\left(40^{\circ}, 45^{\circ}\right)$

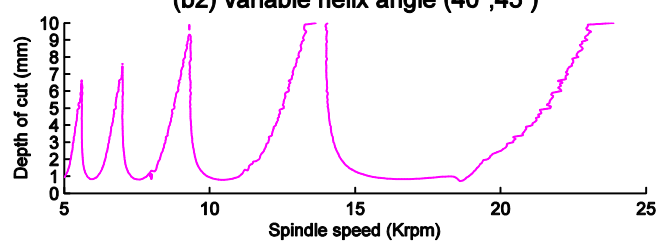

Figure 4. Stability charts for variable helix milling with radial immersion ratio $a_{d c}=0.1$ 


\section{CONCLUSIONS}

In this work, the improved semi-discretization algorithm is proposed for variable helix milling. Due to the variable helix tools the delay between each flute varies along the axial depth of the tool; the tool is discrete into a number of axial layers to solve this problem. Based on Floquet theory, 2-DOF down-milling models are utilized to demonstrate the proposed algorithm. To explore the stability of variable helix milling, uniform helix angle and variable helix angle are compared at two different radial immersion ratios. When the radial immersion ratio adc $=$ 0.05, variable helix milling will lead to stability significantly changed, for example, many unstable islands arose in the Fig .3. And the size of the differences in per tooth's helix angle also has an impact on the stability. However, when the radial immersion ratio adc $=0.1$, variable helix milling has a little effect on the stability.

\section{REFERENCES}

[1] Y. Altintas, "Modeling approaches and software for predicting the performance of milling operations at mal-ubc," Mach Sci Technol, vol. 4, Aug. 2000, pp. 445-478, doi:10.1080/10940340008945718.

[2] Y. Altintas, E. Budak, "Analytical prediction of stability lobes in milling," Cirp Ann-Manuf Techn, vol. 44, 1995, pp. 357-362, doi: 10.1016/S0007-8506(07)62342-7.

[3] Y. Altintas, "Manufacturing Automation: Metal Cutting Mechanics, Machine Tool Vibrations, and CNC Design," Cambridge University Press, 2000.

[4] S. D. Merdol, Y. Altintas, "Multi frequency solution of chatter stability for low immersion milling," J Manuf Sci E-T Asme, vol. 126, Aug. 2004, pp. 459-466, doi:10.1115/1.1765139.
[5] T. Insperger, G. Stepan, "Semi-discretization method for delayed systems," Int J Numer Meth Eng, vol. 55, Oct. 2002, pp. 503-518, doi:10.1002/nme.505.

[6] T. Insperger, G. Stepan, "Updated semi-discretization method for periodic delay-differential equations with discrete delay," Int J Numer Meth Eng, vol. 61 Sep. 2004, pp. 117-141, doi: 10.1002/nme.1061.

[7] P. V. Bayly, J. E. Halley, B. P. Mann, M. A. Davies, Stability of interrupted cutting by temporal finite element analysis, J Manuf Sci E-T Asme, vol. 125 May. 2003, pp. 220-225, doi:10.1115/1.1556860.

[8] Y. Ding, L. Zhu, X. Zhang, H. Ding, "A full-discretization method for prediction of milling stability,” Int J Mach Tool Manu, vol. 50 May. 2010, pp. 502-509, doi:10.1016/j.ijmachtools.2010.01.003.

[9] R. Szalai, G. Stepan, "Lobes and lenses in the stability chart of interrupted turning," J Comput Nonlin Dyn, vol. 1 Jul. 2006, pp. 205-211, doi:10.1115/1.2198216.

[10] M. Zatarain, J. Munoa, G. Peigne, T. Insperger, "Analysis of the influence of mill helix angle on chatter stability," Cirp Ann-Manuf Techn, vol. 55 Aug. 2006, pp. 365-368, doi:10.1016/S00078506(07)60436-3.

[11] T. Insperger, J. Munoa, M. Zatarain, G. Peigne, "Unstable islands in the stability chart of milling processes due to the helix angle," in: CIRP-2 $2^{\text {nd }}$ International Conference on High Performance Cutting (HPC), Vancouver, Canada, 2006.

[12] S. Turner, D. Merdol, Y. Altintas, K. Ridgway, "Modelling of the stability of variable helix end mills," Int J Mach Tool Manu, vol. 47 Jul. 2007, pp. 1410-1416, doi 10.1016/j.ijmachtools.2006.08.028.

[13] N. Sims, B. Mann, S. Huyanan, Analytical prediction of chatter stability for variable pitch and variable helix milling tools, J Sound Vib, vol. 317, Nov. 2008, pp. 664-686, doi: 10.1016/j.jsv.2008.03.045

[14] A. R. Yusoff, N. D. Sims, Optimisation of variable helix tool geometry for regenerative chatter mitigation, Int J Mach Tool Manu, vol. 51, Feb. 2011, pp. 133-141, doi: 10.1016/j.ijmachtools.2010.10.004. 